\title{
Enfoques interaccionales en la lingüística contemporánea: ¿un posible cambio de perspectiva?
}

\section{Interactional approaches in modern linguistics: a possible change of perspective?}

\author{
Marek Baran \\ Uniwersytet Łódzki \\ marek.baran@uni.lodz.pl
}

\begin{abstract}
The study analyses the progressive implementation of interactional approaches in modern linguistics. Starting from the methodological assumptions of the stricto sensu conversational analysis, it is being demonstrated that the approaches initially centred on the reconstruction of the sequential model of the discourse have been opened to the perspectives, which includes not only the interactional modelling of the discursive form, but also the interpersonal construction of meaning. The article also points out the most recent fields of study of interactional linguistics, among them, the analyses dedicated to the socalled conversational negotiations, the theories of verbal politeness or the contrastive and transcultural approaches.
\end{abstract}

Keywords: Interactional Linguistics, Conversational Turn-Taking, Conversational Negotiations, Face-Work

\section{A MODO DE INTRODUCCIÓN}

El propósito del presente estudio es reflexionar acerca de la progresiva implantación de los enfoques interaccionales en la lingüística moderna. Partiendo de los supuestos metodológicos del análisis conversacional stricto sensu, trataré de demostrar en qué medida los planteamientos centrados inicialmente en la reconstrucción del modelo secuencial del discurso, se han ido abriendo a las perspectivas en que contaba no solamente la modelación interaccional de la forma discursiva, sino 
también la construcción interpersonal del sentido. Intentaré señalar, de igual modo, los campos de estudio más recientes de la lingüística interaccional, entre ellos, los análisis dedicados a las así llamadas negociaciones conversacionales, la teoría de cortesía verbal o los acercamientos contrastivos y transculturales. Me acercaré, de igual modo, a los conceptos de intersubjetivización y reflexividad comunicativa cuya operatividad parece ganar cada vez más terreno en los enfoques de corte interaccional. Cabe advertir de inmediato que de ningún modo pretendo trazar la trayectoria histórica o metodológica de distintas corrientes que pertenecen a la denominada lingüística interaccional (tal tarea requeriría un estudio de otras dimensiones, tanto cuantitativas como cualitativas). Mi objetivo se traduce fundamentalmente en el intento de reflejar el cambio de perspectiva, tanto temático como interpretativo, que se está operando dentro de los planteamientos interaccionales.

\section{FORMA - FUNCIÓN - SIGNIFICADO INTERPERSONAL}

Aunque la tradición lingüística desde el principio parecía asumir el carácter polifuncional del habla, durante un lapso de tiempo considerable se juzgó como primordial la función representativa, orientada ésta hacia la expresión de impresiones perceptivas. Además, la tendencia de considerar el discurso individual como la única realidad «observable» y «estudiable», por llamarlo así, dominaba fuertemente a pesar de múltiples declaraciones, también por parte de los lingüistas de orientación estructuralista, sobre el carácter eminentemente social del lenguaje. Dicha preferencia de índole teórico-interpretativa suponía fundamentalmente una percepción sistémica del lenguaje. Como afirma Kerbrat-Orecchioni (2005: 7), citando a Benveniste (1966: 126), la relación «forma - significado», en numerosísimos acercamientos se reducía, en realidad, al estudio de la forma, sin tomar en cuenta el otro correlato, a saber, el significado (a continuación, me esforzaré en demostrar que dicha preferencia por el estudio de la forma, se reflejará también en los análisis de corte interaccional).

El supuesto cambio de perspectiva, que con el tiempo puede observarse en los estudios lingüísticos, parece tener doble cara: la primera remite al interés cada vez más creciente por las interacciones comunicativas como tales (y no tan solo por las posibilidades que ofrece el sistema); la segunda indica que dentro los enfoques interaccionales propiamente dichos, se está también llevando a cabo una modificación notable en cuanto a los campos de análisis, así como a la forma de abordarlos.

$\mathrm{Al}$ tomar como punto de partida el interés por la lengua hablada y al considerar, al mismo tiempo, la conversación como una de las actividades más típicamente humanas (o, en otras palabras, una forma prototípica en que se manifiestan las lenguas, su forma primera de existencia y el modo universal de uso lingüístico (Tusón Valls 2002: 134)), habría, a lo mejor, que mencionar en primer lugar un invento 
técnico que constituyó una verdadera bendición para el desarrollo de los estudios sobre los intercambios conversacionales: se trata, por supuesto, de la grabadora. Hasta entonces, la recogida de datos se presentaba como extremadamente complicada, parando así los análisis de las interacciones verbales reales. Sin duda ninguna, la aparición del magnetófono tuvo un impacto comparable a la aplicación del microscopio en otros campos de ciencia. Sin embargo, sería erróneo pensar que la «bendita grabadora» cambió por sí sola el panorama de los estudios lingüísticos. Si Wiliam Labov (1972) en los años setenta emite una apasionado llamamiento a la práctica de una lingüística que se apoye ante todo en el lenguaje que emplean realmente los interlocutores nativos entre sí en sus comunicaciones diarias, lo hace porque es perfectamente consciente del predominio de la lingüística que los francófonos denominan linguistique de chambre (lingüística practicada en los despachos, la que se opone a la lingüística de campo). Durante mucho tiempo, las conversaciones cotidianas se consideran como simplemente triviales, lo que desanima a los potenciales investigadores a tomarlas como objeto de análisis. La supuesta banalidad de las conversaciones coloquiales se une, curiosamente, al presentimiento, por no decir, a la conciencia de que éstas constituyen una materia extremadamente compleja, o incluso «anárquica», que no se deja reducir al sistema de oposiciones binarias tan queridas por los estructuralistas. Dicha complejidad junto con el escaso prestigio del que gozaban los análisis interaccionales paran indudablemente el interés por dicha materia durante un tiempo considerable, ante todo si tomamos en cuenta el contexto lingüístico de la Romania, fuertemente marcado por el legado sausserano $^{1}$. Si hacemos abstracción de esta última, veremos un creciente interés por el interaccionismo del otro lado del Océano, donde, en los años treinta, en el marco de los estudios de corte sociológico, se va desarrollando el interaccionismo simbólico, cuyo seguidores serán tanto E. Goffman como H. Sacks y E. Schegloff, considerados a menudo como fundadores del análisis conversacional. No es de extrañar que los estudios interaccionales encuentren un terreno propicio inicialmente en los Estados Unidos: allí, una riquísima tradición investigadora orientada hacia las relaciones entre la lengua y la cultura se reflejaba siempre en la importancia otorgada a la antropología, la etnología como la etnografía (también la etnografía de la comunicación desarrollada luego a partir de los años sesenta $)^{2}$.

\footnotetext{
${ }^{1}$ Aunque el Cours de linguistique générale afirmaba la primacía de lo oral, así como el carácter social del lenguaje, los análisis que se inscribían en la línea estructuralista reducían muy a menudo la lengua a un sistema descontextualizado, interesándose, además, tan solo por sus realizaciones escritas (Kerbrat-Orecchioni 2005: 10).

${ }^{2}$ Como observa justamente Kerbrat-Orecchioni (2005: 11), los estudios etnológicos en sus inicios constituían una especie de «etnología de otra parte». Los investigadores estadounidenses se interesaban durante un tiempo considerable casi únicamente por las sociedades «exóticas» o «lejanas», para llamarlas de otro modo. La mirada lanzada hacia distintas formas bajo las que se manifiesta la comunicación interpersonal fue, no obstante, la más importante; además, con el tiempo «la etnología de otra
} 


\section{ANÁLISIS CONVERSACIONAL: DEL MODELO SECUENCIAL DEL HABLA A LA GESTIÓN INTERPERSONAL DEL DISCURSO}

Si quisiéramos indicar otro factor que tuvo un impacto comparable al de la invención de la grabadora, deberíamos indudablemente remitirnos a la constitución de los grandes córpora de lengua hablada. La lingüística de corpus permitió el auge del análisis conversacional (ing. conversation analysis), que parece marcar un antes y un después en los planteamientos lingüísticos de corte interaccional. Como es bien sabido, el análisis conversacional se interesó de modo particular por los procedimientos que utilizan los miembros de una sociedad dada para gestionar de forma adecuada el conjunto de problemas comunicativos a los que están confrontados en la vida diaria. La conversación, desde el primer momento, se interpretó como una de las formas fundamentales de construcción y organización social. Se la presentaba como objeto de estudio y como muestra de construcción de la realidad debido a su carácter interactivo. Entre uno de los fundamentos de los planteamientos del análisis conversacional se encontraba la convicción de que cualquier enunciado, sea cual sea su grado de complejidad, no aparece nunca como resultado de un solo hablante: es siempre fruto de un proceso interactivo, por tanto, el modelo interpretativo no puede centrarse únicamente en la producción de uno de los interlocutores. La dualidad de los hablantes, el nivel de encadenamiento de los enunciados en un orden secuencial junto a la coordinación de las acciones son otros elementos que ayudan a entender la actividad verbal (Baylon, Mignot 1999: 264). El análisis conversacional, al ser fiel a los fundamentos de la etnometodología, consideraba que la realidad social nunca se da de modo objetivo: la están creando constantemente los agentes sociales a lo largo de sus interacciones. La actividad de los cooperantes en la comunicación se presenta, bajo esta óptica, como apenas determinada por normas o conceptos exteriores: su significación reside básicamente en el trabajo de los interlocutores, quienes definen y redefinen constantemente las reglas ${ }^{3}$. La lingüística interaccional, que continúa y desarrolla la labor emprendida por los partidarios del análisis conversacional, reconoce a la interacción un papel constitutivo no solo de las prácticas de los hablantes sino también de la estructuración de los recursos lingüísticos. Según

parte» se vio complementada por «la etnología de aquí», es decir, por la etnología de las sociedades indoeuropeas.

${ }^{3}$ Los etnógrafos de la comunicación, por su parte, acentuaban el papel de las convenciones o condicionamientos sociales preestablecidos en una comunidad de habla dada. Se trata, de hecho, de dos puntos de mira que suponen dos concepciones distintas del papel de los recursos lingüísticos en la interacción. Si el análisis conversacional pone de manifiesto que las formas lingüísticas no se explotan solo interaccionalmente sino que se hallan configuradas por la interacción (los hablantes estructuran los recursos de la lengua, imponiéndoles modos de organización y valores situados) (Mondada 2001: 82), la etnografía de la comunicación formula la hipótesis según la cual la organización de la interacción explota al máximo los recursos lingüísticos según sus características formales y sus peculiaridades socioculturales. 
los principios metodológicos de dichos planteamientos, se procede al establecimiento de grandes córpora de datos orales auténticos ${ }^{4}$ que deberían permitir el descubrimiento de las regularidades en la utilización de unas determinadas estructuras en ciertos contextos y hacer observables los procesos de coordinación, sincronización y articulación ordenada de las actividades (Mondada 2001: 65). Una observación minuciosa de la gestión comunicativa que se da en las interacciones lleva a la delimitación de lo que Kerbrat-Orecchioni (1996: 14) denomina como tecnología de la conversación: el foco de atención se dirige hacia las técnicas que los hablantes utilizan cumpliendo con ciertas obligaciones que les impone la situación y el contexto comunicativos. Así pues, resulta posible reconstruir los modos en los que los interlocutores aseguran, por ejemplo, la alternancia de los turnos de habla, llevan a cabo una negociación, estructuran un relato o reparan ciertas deficiencias que pueden producirse a lo largo del acto comunicativo.

Conviene subrayar que la problemática de alternancia de turnos constituyó una preocupación mayor de los partidarios del análisis conversacional. La reconstrucción del modelo secuencial de los turnos condujo a una especie de formalización de la base organizativa de la conversación espontánea. Este nivel sistémico de análisis ha sido desarrollado especialmente por Sacks, Schegloff \& Jefferson (1974) (aunque ya Goffman (1971) señalaba que la conversación cotidiana requería un análisis que atendiera a la organización y la gestión de los turnos), quienes observaron determinadas regularidades en la forma de coordinar el sistema de turnos 5 .

\footnotetext{
${ }^{4}$ La recolección de datos se lleva a cabo buscando una invasión mínima en la actividades registradas y una integración máxima de quien investiga en los grupos observados.

${ }^{5}$ Entre dichas regularidades, hay catorce rasgos fundamentales por los que, según Sacks, Schegloff \& Jefferson (1974), destacan las interacciones espontáneas. Las reproduzco aprovechando la traducción española ofrecida por Tusón Valls (2002: 137):

1. El cambio de hablante es recurrente o, al menos, se produce. Es decir, una de las características de la conversación es que es dialogal.

2. En general, no habla más de una persona a la vez.

3. Los solapamientos (dos - o más - participantes a la vez) son comunes pero breves.

4. Las transiciones más comunes entre un turno de palabra y el siguiente son las que se producen sin intervalos ni solapamientos, o las que se producen con un breve intervalo.

5. El orden de los turnos de palabra no es fijo.

6. La duración de los turnos de palabra no es fija, si bien se tiende a un cierto equilibrio.

7. La duración de una conversación no se especifica previamente.

8. Lo que dicen los hablantes no se ha especificado previamente.

9. La distribución de los turnos de palabra no se ha especificado previamente.

10. El número de hablantes puede variar.

11. El discurso puede ser continuo o discontinuo.

12. Existen técnicas para la distribución de los turnos.

13. Se utilizan diferentes unidades formales de construcción de los turnos (una palabra, una frase, una oración, etc.).

14. Existen mecanismos para reparar los errores o las transgresiones en la toma de la palabra.
} 
El estudio y la descripción de los mecanismos de heteroselección o de autoselección que regían la mecánica conversacional fue, sin duda ninguna, un importante aporte del análisis conversacional. Deberíamos, no obstante, ser conscientes de que la detenida observación de la lógica secuencial de la alternancia de los turnos supuso tanto un innegable aporte como una supuesta limitación del enfoque al que me estoy refiriendo. Utilizo el término de limitación dado que, en algunas ocasiones, el análisis de la alternancia de turnos, propio de lo que Kerbrat-Orecchioni (2005) llama con elegancia «discurso en interacción», no dejaba sitio al estudio de otros fenómenos comunicativo-interaccionales. Así pues, se podía tener la impresión de que la alternancia de turnos constituía una especie de «fin en sí», sin ser realmente considerada lo que es, o sea, un medio al servicio de la construcción del sentido, de la circulación de la información, del mantenimiento de la relación social, o de tantísimas cosas más (Kerbrat-Orecchioni 2005: 6). En las reelaboraciones del modelo, o dicho con más exactitud, en los planteamientos que suceden al análisis conversacional y que hoy en día suelen considerarse como enfoques interaccionales, los turnos empiezan a verse de un modo diferente. No puede ser de otra manera si admitimos que la actividad verbal real no puede limitarse a la construcción de los turnos de habla. Además, convendría preguntarse ¿qué son, en realidad, las secuencias en que se organizan los intercambios conversacionales? Al optar por un planteamiento de corte comunicativo habría que afirmar que estamos básicamente ante actos de habla que se definen por sus propiedades semántico-pragmáticas. Sin lugar a dudas, la forma secuencial de los intercambios estructura la materia discursiva dándole cohesión y coherencia, pero, al fin y al cabo, dicha materia no se reduce tan solo a su aspecto formal. En cada intercambio comunicativo, se pone en marcha un determinado contenido, un contenido que se construye paso a paso entre los interlocutores, de acuerdo con determinadas finalidades que estos últimos se proponen y en función de distintos incidentes interaccionales que, de un modo más o menos imprevisto, suceden a lo largo de las interacciones. Así, llegamos a otra faceta de la lingüística interaccional actual, a saber, la que toma en cuenta un sinfín de nociones y conceptos operativos en las disciplinas afines. Pienso, desde luego, en las nociones de pragmática y sociolingüística ${ }^{6}$ tales como: «imagen», «territorio», «ritual comunicativo» (cf. Goffman 1967), «competencia comunicativa», «evento comunicativo» (cf. Gumperz 1982; Hymes 1967, 1971, 1972), «contextualización», «inferencia» (cf. Gumperz 1982), «actos de habla» (cf. Austin 1962; Searle 1969), «principio de cooperación» e «implicatura» (cf. Grice 1975), «face-work», «cortesía positiva vs cortesía negativa», «acto amenazador de imagen» (Brown y Levinson 1978, 1987). La «materia discursiva» percibida como en términos interaccionales abarca, además, no solamente las conversaciones, sino también otros eventos marcados por un sistema de influencias mutuas que se registran entre los interlocutores (así, por ejemplo, los

\footnotetext{
${ }^{6}$ Algunos de ellos presentes ya en los inicios de los enfoques de índole interaccional.
} 
chats caben perfectamente en el marco interaccional). Notemos a este respecto que aunque toda enunciación lleva marcas interactivas (el discurso que se percibe en términos generales normalmente conlleva elementos de dialogismo), la noción de interacción operativa en los enfoques a los que nos estamos refiriendo supone que el destinatario puede influenciar o modificar el comportamiento del emisor de un modo imprevisible, casi "brusco", mientras este último está todavía "construyendo" su discurso. En otras palabras, para que haya interacción es imprescindible poder observar algunos fenómenos de retroacción inmediata, o, como se los denomina en los estudios interaccionales, fenómenos de reflexividad ${ }^{7}$ (Kerbrat-Orecchioni 2005: 17). En este sentido, «el discurso en interacción» como objeto de estudio excluye los discurso monologales en los que el destinatario está ausente. Podría, tal vez, hablarse incluso de diferentes grados de interactividad, determinando, por ejemplo, la naturaleza de las participaciones mutuas de los interlocutores, la dinámica de la alternancia de los turnos de habla o el involucramiento de los participantes en la interacción.

\section{NEGOCIACIONES CONVERSACIONALES E INTERACCIONALES}

Como estamos viendo, el cambio de perspectiva anunciado en el título del presente estudio, se traduce, entre otros, en la ampliación del campo de estudio. La conversación sigue, obviamente, considerándose como la forma más básica de la actividad lingüística interaccional (una especie de sistema social en miniatura, como lo quería Goffman 1973: 21), pero el análisis del discurso en interacción incluye otras formas de interacciones comunicativas (entre ellas, por ejemplo, las interacciones mediáticas, las que se registran en los comercios y los servicios, las interacciones en la consulta médica o las interacciones en otros contextos institucionales, como, por ejemplo, el contexto escolar o jurídico [para servirse de los ámbitos ya estudiados por los lingüistas interaccionistas] $)^{8}$. El contexto de análisis así ampliado nos lleva, por su parte, a uno de los fenómenos clave de la lingüística interaccional moderna, a saber, la cuestión de las negociaciones conversacionales. La noción de negociación interaccional puede remitir, en este caso, a una doble realidad. Por una parte, se la

\footnotetext{
${ }^{7}$ Obviamente, en un sentido diferente del propio de las ciencias exactas en las que la «reflexividad» remite a la relación que un elemento dado mantiene con sí mismo. La «reflexividad interaccional» significa siempre una relación que se da entre dos (o más) interlocutores que se influencian mutuamente a lo largo de los intercambios que entretienen.

${ }^{8}$ La lingüística interaccional se dedica en este caso, entre otros, a la delimitación de los scripts, es decir, un tipo de guiones según los que se realizan los intercambios en unos determinados ámbitos (véase, en este sentido, por ejemplo, los análisis de las «interactions scryptées» realizados por KerbratOrecchioni $(2003,2004,2004 b)$ o Traverso $(2000,2004)$. Se estudian, de igual modo, aspectos como: temas de los intercambios (topics en la terminología interaccional anglosajona), su extensión y duración, géneros interaccionales empleados, tipos de los cambios interruptivos (véase Baran 2015).
} 
reserva a los casos en que inicialmente se da algún conflicto que los interlocutores irán gestionando; por otra, algunos investigadores entienden por negociación todo tipo de construcción o cooperación interlocutiva. Para poner un ejemplo, podría servirme de un mecanismo ampliamente estudiado por los lingüistas (ante todo, los sociolingüistas), pero reinterpretado de alguna manera por la lingüística de índole interaccional. Pienso, en concreto, en las formas pronominales de tratamiento, tradicionalmente estudiadas a la luz de la dicotomía poder // solidaridad (cf. Brown y Gilman 1960). Como demuestra Blas Arroyo (2005), los interlocutores recurren al llamado uso negociado de dichas formas, reestructurando así el marco participativo inicial. Numerosas formas lingüísticas, entre ellas las mencionadas formas de tratamiento, se convierten, pues, en indicios de contextualización (noción empleada por Gumperz 1982), esto es, en "marcas semióticas que permiten renegociar los papeles sociales desempeñados por los participantes en el curso de la interacción"9 (Blas Arroyo 2005: 316). En el caso de las formas de tratamiento utilizadas en el español peninsular, pueden registrarse unas llamativas secuencias interactivas en las que destaca el tránsito progresivo que se produce entre diversos sistemas de tratamiento $\left[\text { usted - usted } \rightarrow \text { usted }-t_{u} \rightarrow t u ́ \text { - tú }\right]^{10}$ (Blas Arroyo 1994: 403-409). Así pues, retomando el concepto de negociación conversacional, deberíamos tomar conciencia de que en el caso de la comunidad hispanohablante, la transición del tratamiento formal al informal se realiza en numerosísimas ocasiones de manera paulatina, a base de un acuerdo tácito.

El ejemplo citado supra da cuenta de otro aspecto clave característico de los enfoques interaccionales modernos. Se trata, de hecho, su faceta comparativa o contrastiva. En realidad, los estudios interaccionales contemporáneos raras veces se llevan a cabo haciendo abstracción de la observación del uso comunicativo propio de otras comunidades de habla. Se busca, de este modo, una especie de tertium comparationis constante, el cual relativiza el uso de los recursos y mecanismos propios de una sociedad dada. De ese modo, se pretende huir del «pecado original» de muchos acercamientos etnolingüísticos iniciales que caían a menudo en unas consideraciones excesivamente etnocentristas o unidireccionales. La perspectiva comparativa o contrastiva, por no decir transcultural, propia de la lingüística interaccional actual permite varias reinterpretaciones de determinados puntos de teorías pragmáticas o sociolingüísticas fuertemente arraigadas en la lingüística como tal. Para poner otro ejemplo, podría recurrirse a la clasificación de los actos de habla. La conocidísima categorización de Searle se ve así reinterpretada o, por decirlo de otro modo,

\footnotetext{
${ }^{9}$ Se trata básicamente de marcas lingüísticas y no lingüísticas que relacionan el mensaje con el conocimiento contextual que los hablantes poseen.

${ }^{10}$ En cambio, otras comunidades de habla, como, por ejemplo, la polaca o la alemana, optan por un guion diferente de acuerdo con el que la forma de trato se establece a través de un ritual explícito (en polaco: Czy możemy sobie mówić na ty? [¿Podemos tratarnos de tú?]).
} 
complementada por la tipología que toma en cuenta ante todo la variabilidad interlingüística. Así pues, en base a la selección de unos determinados actos de habla y la comparación de su funcionamiento en dos o más comunidades lingüísticas suelen delimitarse $^{11}$ : - actos de habla que existen en unas lenguas y no se registran en otras, - actos de habla existentes en diferentes lenguas pero asociados de modos distintos, - actos de habla existentes en dos (o más) lenguas pero utilizados en contextos distintos y con finalidades diferentes, - actos de habla que difieren en cuanto a su realización lingüístico-discursiva, - actos de habla coincidentes desde el punto de vista formal pero empleados con frecuencias distintas, - actos de habla estrechamente vinculados con conocimientos socioculturales previos.

\section{A MODO DE CONCLUSIÓN}

Una progresiva reconceptualización de distintos aspectos formales y funcionales de los intercambios verbales conduce indudablemente a una ampliación de la perspectiva de análisis de lo que hoy se conoce como interaccionismo lingüístico. Lo formal, privilegiado en los acercamientos analíticos iniciales, empieza así a verse también a través de las interpretaciones funcionales e interpersonales que acentúan las características de índole intencional. Las intencionalidades comunicativas suelen estudiarse, por su parte, en el marco de unos determinados guiones (scripts) percibidos fundamentalmente en términos de negociaciones conversacionales. Estas últimas se someten a estudio teniendo en cuenta los condicionamientos de carácter sociocultural y sociopragmático (las visiones unidireccionales marcadas por un fuerte etnocentrismo dejan paso a los planteamientos de índole transcultural). En este sentido es también la cortesía verbal la que se contempla desde una óptica que une la supuesta universalidad del principio de comportamiento cortés con la nouniversalidad de los mecanismos por medio de los que se llevan sus determinadas estrategias. La cortesía que rige el denominado face-work depende, así, de las codificaciones sociopragmáticas de la interacción social, codificaciones que pueden diferir en distintas comunidades de habla.

Conviene subrayar, además, que el cambio de perspectiva que se deja observar en los enfoques interaccionales actuales no remite únicamente al tipo de intercambios comunicativos analizados o al estudio de diferentes clases de operadores interaccionales. Se trata, de igual modo, de la relación que los planteamientos interaccionales mantienen con los enfoques (no)-normativos. El interaccionismo lingüístico, al servirse de grandes córpora de datos orales, permite "pesar", para emplear un giro metafórico, la verdadera representatividad de diferentes formas, o recursos lingüísticos. No en pocas ocasiones ocurre que empleos considerados a menudo como

\footnotetext{
${ }^{11}$ Recurro a la propuesta clasificatoria de Miquel (2004).
} 
«atípicos» o incluso «agramaticales» empiezan a reconsiderarse a través de los conceptos pragmáticos o justamente interaccionales. «Lo atípico» o «lo agramatical» empieza a verse así como un fenómeno que, tal vez, esconde determinadas finalidades comunicativas o intercomunicativas. En el cruce de «lo gramatical», «lo pragmático» $\mathrm{y}$ «lo interaccional», resultan verdaderamente sugerentes los estudios que últimamente se realizan sobre distintas modalidades hispanoamericanas (a veces influenciadas por las lenguas indígenas). La clásica ya noción de modalidad encuentra de este modo un apoyo de tipo cuantitativo y cualitativo en los estudios que, como he apuntado, basándose en los córpora estudian detenidamente contextos interaccionales reales. Distintos fenómenos comunicativos se ven así bajo el prisma de (inter)subjetivización, concebida esta como proceso que conduce a la aparición de marcadores discursivos con matices significativos diversos que permiten a los hablantes involucrarse en los eventos comunicativos, codificando al mismo tiempo su punto de vista sobre ellos ${ }^{12}$. Se trata indudablemente de una vía interpretativa digna de ser desarrollada en los análisis futuros.

${ }^{12}$ A modo de ejemplo podrían citarse los trabajos de Risco $(2014,2018)$ sobre el doble posesivo o el doble adverbio negativo en el español andino. Como demuestra la investigadora, la variación morfosintáctica observada en distintas modalidades sudamericanas del español en numerosas ocasiones se consolida a partir de las motivaciones que llevan a un hablante a manifestar diferentes perspectivas frente a la misma escena. 


\section{BIBLIOGRAFIA}

Austin, J.L. (1962). How To Do Things With Words. Oxford: Clarendon Press.

Baran, M. (2015). Armonía y contraste en los sistemas de alternancia de turnos de habla. In J.M.

Santos Rovira (ed.), Armonía y contrastes. Estudios sobre la variación dialectal, histórica y sociolingüistica de español (pp. 289-298), Lugo: Editorial.

Baylon, Ch., Mignot, X. (1999). La communication. Paris: Nathan.

Benveniste, É. (1966). Problèmes de linguistique générale, t. I. Paris: Gallimard.

Blas Arroyo, J.L. (1994). De nuevo sobre el poder y la solidaridad. Apuntes para un análisis interaccional de la alternancia. Tú/Usted. Nueva Revista de Filología Hispánica, XLII/2, $385-414$.

Blas Arroyo, J.L. (2005). Sociolingüística del español. Desarrollos y perspectivas en el estudio de la lengua española en contexto social. Madrid: Cátedra.

Brown, P., Levinson, S. (1978). Universals in language usage. Politeness phenomena. In E. Goody (ed.), Questions and Politeness. Strategies in Social Interaction (pp. 56-289). Cambridge: Cambridge University Press.

Brown, P., Levinson, S. (1987). Politeness. Some Universals in Language Usage. Cambridge: Cambridge University Press.

Brown, R., Gilman, A. (1960). The pronouns of power and solidarity. In T.A. Sebeok (ed.), Style in Language (pp. 253-276). New York: Wiley.

Goffman, E. (1967). Interaction Ritual. Essays on Face-to-Face Behavior. New York: Doubleday.

Goffman, E. (1971). Relations in Public. Microstudies of the Public Order. New York: Basic Books.

Goffman, E. (1973). La mise en scène de la vie quotidienne. 1 - La présentation de soi. Paris: Minuit.

Grice, H.P. (1975). Logic and conversation. In P. Cole \& J.L. Morgan (eds.), Syntax and Semantics 3: Speech Acts (pp. 41-58). New York: Academic Press.

Gumperz, J. (1982). Discourse Strategies. Cambridge: Cambridge University Press.

Hymes, D. (1967). Models of the Interaction of Language and Social Setting. Journal of Social Issues, 23/2, 8-28.

Hymes, D. (1971). Competence and performance in linguistic theory. In R. Huxley, E. Ingram (eds.), Language Acquisition. Models and Methods (pp. 3-28). London: Academic Press.

Hymes, D. (1972). Models of the interaction of language and social life. In J.J. Gumperz \& D.H. Hymes (eds.), Directions in Sociolinguistics. The Ethnography of Communication (pp. 35-71). New York: Holt, Rinehart, Winston.

Kerbrat-Orecchioni, C. (1996). La conversation. Paris: Seuil.

Kerbrat-Orecchioni, C. (2003). Les récits conversationnels, ou la parole "ordinaire", c'est tout un art. In J.-B. Martin \& N. Decourt (eds.), Littérature orale, paroles vivantes et mouvantes (pp. 99-121). Lyon: PUL.

Kerbrat-Orecchioni, C. (2004a). Analyse des conversations et négociations conversationnelles. In M. Grosjean \& M. Mondada (éds.), La négociation au travail (pp. 18-41). Lyon: PUL.

Kerbrat-Orecchioni, C. (2004b). Que peut-on faire avec du dire. Cahiers de Linguistique Française, 26, 27-43.

Kerbrat-Orecchioni, C. (2005). Le discours en interaction. Paris: Armand Colin. 
Labov, W. (1972). Sociolinguistic Patterns. Philadelphia: Pennsylvania Press.

Miquel, L. (2004). Lengua y cultura desde una perspectiva pragmática: algunos ejemplos aplicados al español. redELE, 2, 1-25.

Mondada, L. (2001). Por una lingüística interaccional. Revista iberoamericana de Discurso y Sociedad, 3 (3), 61-89.

Risco, R. (2014). Español andino: perspectiva de mundo en la variación interhablante. In: J. Wilk-Racięska, A. Nowakowska-Głuszak \& C. Tatoj (eds.), Encuentros entre lenguas, literaturas y culturas de los territorios luso-hispanos. Perspectivas diferentes (pp. 117-144). Katowice: Wydawnictwo Uniwersytetu Śląskiego.

Risco, R. (ed.) (2018). Estudios de variación y contacto lingüistico en el español peruano. La Plata: Universidad Nacional de la Plata.

Sacks, H., Schegloff, E., Jefferson, G. (1974). The Simplest Systematics for the Organization of Turn-Taking in Conversation. Language, 50, 696-731.

Searle, J.R. (1969). Speech Acts. Cambridge: Cambridge University Press.

Traverso, V. (2000). Autour de la mise en œuvre d'une comparaison interculturelle. L'exemple des comportements confirmatifs dans des émissions radiophoniques françaises et syriennes. In V. Traverso (ed.), Perspectives culturelles sur l'interaction (pp. 33-51). Lyon: PUL.

Traverso, V. (2004). Cristallisation des désaccords et mise en place de négociations dans l'interaction: des variations situationnelles. In M. Grosjean \& L. Mondada (eds.), La négociation au travail (pp. 43-68). Lyon: PUL.

Tusón Valls, A. (2002). El análisis de la conversación: entre la estructura y el sentido. Estudios de Sociolingüistica, 3 (1), 133-153. 